\title{
Avaliação agronômica da rebrota, dinâmica de decomposição e liberação de nutrientes de flemingia (Flemingia macrophylla (Willd.) Kuntze ex Merr.) ${ }^{1}$
}

\author{
Alexandre Porto Salmi², José Guilherme Marinho Guerra³, Antônio Carlos de Souza Abboud², \\ Murilo Gonçalves Júnior ${ }^{2}$
}

\section{RESUMO}

Flemingia macrophylla é uma leguminosa arbustiva, de origem asiática, pouco explorada no Brasil. Em vista disso, este estudo teve como objetivo avaliar a biomassa da rebrota da espécie, a acumulação de nitrogênio na parte aérea e a decomposição e liberação de nutrientes de folhas e caules de flemingia. Para ambos os experimentos, o delineamento adotado foi em blocos casualizados, com quatro repetições. A espécie apresenta excelente capacidade de rebrotar, sendo que os cortes executados a 1,2 m do nível do solo produziram mais de $34 \mathrm{Mg}$ de matéria seca em nove cortes, realizados durante o experimento, equivalendo a $804 \mathrm{~kg} \mathrm{~N} \mathrm{ha}^{-1}$, superando os demais tratamentos, que consistiam em cortes a 0,6 m de altura que produziram $29 \mathrm{Mg} \mathrm{MS} \mathrm{ha}^{-1}$, acumulando $691 \mathrm{~kg} \mathrm{~N} \mathrm{ha}^{-1}$, seguido dos cortes a 0,3 m com 18

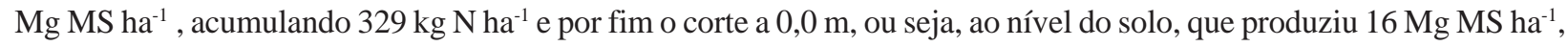
com acúmulo de $211 \mathrm{~kg} \mathrm{~N} \mathrm{ha}^{-1}$. O tempo de meia vida $\left(\mathrm{T}_{1 / 2}\right)$ da decomposição foi de 91, 95 e 97 dias para folha, caule, e, caule mais folha, respectivamente. A liberação de N, P, Ca e Mg seguiu o mesmo padrão da decomposição; já o K foi liberado em taxa mais acelerada.

Palavras-chave: leguminosa, rebrota, tempo de meia vida.

\section{ABSTRACT}

\section{Regrowth, dynamics of decomposition and release of nutrients by flemingia (Flemingia macrophylla (Willd.) Kuntze ex Merr.)}

Flemingia macrophylla is a shrubby legume of Asian origin, little explored in Brazil. The objectives of this study were the evaluation of the regrowth biomass of the species, accumulation of nitrogen in the air and evaluation of decomposition and release of nutrients from the aerial part of flemingia. Both experiments were arranged in a randomized complete block design with four replications. The regrowth biomass estimation of flemingia was performed after cutting at different heights above ground level $(0.0 \mathrm{~m} ; 0.3 \mathrm{~m} ; 0.6 \mathrm{~m} ; 1.2 \mathrm{~m})$. The highest cutting height $(1.2 \mathrm{~m})$ maximized biomass production, at all cutting dates. Up to $34 \mathrm{Mg} \mathrm{DM} \mathrm{ha}^{-1}$ were measured in nine cuttings, at $1.2 \mathrm{~m}$ cutting height, the equivalent to $804 \mathrm{~kg} \mathrm{~N} \mathrm{ha}^{-1}$, outperforming the $0.6 \mathrm{~m}$ cutting which yielded $29 \mathrm{Mg} \mathrm{DM} \mathrm{ha}^{-1}$ and $691 \mathrm{~kg} \mathrm{~N}^{-1}$; the 0.3 m cutting treatment yielded $18 \mathrm{Mg} \mathrm{DM} \mathrm{ha}^{-1}$ and accumulated $329 \mathrm{~kg} \mathrm{~N} \mathrm{ha}^{-1}$; finally, cutting at ground level, yielded 16 $\mathrm{Mg} \mathrm{DM} \mathrm{ha}{ }^{-1}$ with accumulation of $211 \mathrm{~kg} \mathrm{~N} \mathrm{ha}^{-1}$. The half-life time $\left(\mathrm{T}_{1 / 2}\right)$ of the decomposition was 91,95 and 97 days for leaves, stems and stems + leaves, respectively. The release of $\mathrm{N}, \mathrm{P}, \mathrm{Ca}$ and $\mathrm{Mg}$ followed the same pattern of decomposition, however $\mathrm{K}$ was released in a more accelerated rate.

Key words: legume, regrowth, half-life time.

Recebido para publicação em 10/04/2013 e aprovado em 06/05/2013.

'Parte da Tese de Doutorado do primeiro autor no curso de Pós-Graduação em Fitotecnia da UFRRJ. Apoio financeiro Fundação Carlos Chagas de Amparo à Pesquisa do Estado do Rio de Janeiro (FAPERJ)

2Engenheiro-Agrônomo, Doutor. Departamento de Fitotecnia, Universidade Federal Rural do Rio de Janeiro, BR 465 Km 7, Seropédica, Rio de Janeiro, Brasil. salmi@ ufrrj.br (autor para correspondência); abboud@ufrri.br; murilojr@yahoo.com.br

${ }^{3}$ Engenheiro-Agrônomo. Embrapa Agrobiologia, BR 465 Km 7, 23890-000, Seropédica, Rio de Janeiro, Braasil. gmguerra@cnpab.embrapa.br 


\section{INTRODUÇÃO}

A espécie Flemingia macrophylla tem-se mostrado excelente alternativa de leguminosa para inclusão em diversos sistemas de cultivo, para servir como adubo verde. Seu centro de origem é o sudeste asiático, tem porte arbustivo com muitas ramificações e hábito perene, com excelente capacidade de produção de biomassa (Andersson et al., 2006). Essas características são desejáveis nos sistema de consórcio, na forma de aleias, ou seja, o plantio de faixas intercalares de leguminosas, que são podadas periodicamente, no intuito de favorecer o desempenho das culturas consorciadas pela decomposição da biomassa da poda.

A prática de adubação verde tende a minimizar o uso de insumos de fora da propriedade, resultando em vantagem econômica, especialmente para agricultores com menos recursos. Com a crescente demanda de tecnologias que utilizam insumos biológicos, com recursos locais, passou a ser ainda mais importante conhecer a produtividade de biomassa e a dinâmica de decomposição e a de liberação de diversos nutrientes ao solo. Dessa forma, é possível adequar o manejo da leguminosa, a fim de sincronizar a disponibilidade de nutrientes com a necessidade da cultura consorciada (Costa et al., 2012), uma vez que, a espécie apresenta características desejáveis como adubo verde.

Face ao exposto, este trabalho tem como objetivo quantificar a biomassa da rebrota e o acúmulo de nitrogênio, em diferentes épocas e alturas de corte, avaliar a dinâmica de decomposição e a de liberação dos nutrientes $\mathrm{N}, \mathrm{P}, \mathrm{K}, \mathrm{Ca}$ e $\mathrm{Mg}$, do resíduo vegetal da parte aérea da espécie Flemingia macrophylla.

\section{MATERIAL E MÉTODOS}

O experimento foi realizado na Embrapa Agrobiologia, em Seropédica-RJ ( $22^{\circ} 45^{\prime} \mathrm{S}$ e $43^{\circ} 42^{\prime} \mathrm{W}$ e altitude de $\left.33 \mathrm{~m}\right)$. O clima da região é da classe Aw, segundo Köppen, apresentando inverno pouco rigoroso e seco, com chuvas frequentes, durante o verão. A semeadura foi realizada em outubro de 2006, sob casa de vegetação, em bandejas de isopor de 72 células, com duas sementes por célula; procedeu-se à irrigação por microaspersores e as mudas foram irrigadas duas vezes ao dia. Efetuou-se a quebra da dormência das sementes com o tratamento pré-germinativo de imersão em água morna (Salmi et al., 2008). As mudas foram plantadas no dia 12 de dezembro de 2006, espaçadas de $2,0 \mathrm{~m} \times 0,5 \mathrm{~m}$, formando um talhão de $34 \mathrm{~m}$ x $32 \mathrm{~m}$, em uma área total de $1088 \mathrm{~m}^{2} \mathrm{e}$ área útil de $630 \mathrm{~m}^{2}$, em um Planossolo Háplico (Embrapa, 2006). A análise da terra apresentou, na camada de $0-20 \mathrm{~cm}$, as seguintes características químicas: $12,9 \mathrm{~g} \mathrm{~kg}^{-1} \mathrm{de} \mathrm{C}, \mathrm{pH}$ em água 5,6 , $1,3 \mathrm{cmol}_{\mathrm{c}} \mathrm{dm}^{-3} \mathrm{de} \mathrm{Ca}, 1,0 \mathrm{cmol}_{\mathrm{c}} \mathrm{dm}^{-3} \mathrm{de} \mathrm{Mg}, 0 \mathrm{cmol}_{\mathrm{c}} \mathrm{dm}^{-3}$ de Al, 0,37 $\mathrm{cmol}_{\mathrm{c}} \mathrm{dm}^{-3} \mathrm{de} \mathrm{K}, 5 \mathrm{mg} \mathrm{dm}^{-3}$ de Pdisponível e na camada de 20-40 cm: 7,9 $\mathrm{g} \mathrm{kg}^{-1}$ de $\mathrm{C}, \mathrm{pH}$ em água 5,3, $0,9 \mathrm{cmol}_{\mathrm{c}} \mathrm{dm}^{-3} \mathrm{de} \mathrm{Ca}, 0,7 \mathrm{cmol}_{\mathrm{c}} \mathrm{dm}^{-3} \mathrm{de} \mathrm{Mg}, 0,3 \mathrm{cmol}_{\mathrm{c}} \mathrm{dm}^{-3}$ de $\mathrm{Al}, 0,07 \mathrm{cmol}_{\mathrm{c}} \mathrm{dm}^{-3} \mathrm{de} \mathrm{K}, 3 \mathrm{mg} \mathrm{dm}{ }^{-3}$ de $\mathrm{P}$ disponível. Com base na análise da terra, a adubação realizada no plantio foi de $60 \mathrm{~kg}$ de $\mathrm{P}_{2} \mathrm{O}_{5}$ ha $^{-1}$ e $40 \mathrm{~kg}$ de $\mathrm{K}_{2} 0 \mathrm{ha}^{-1}$.

Para a avaliação do potencial da rebrota, realizou-se um primeiro corte, em janeiro de 2008, para estabelecimento das alturas de corte, em plantas com 365 dias de desenvolvimento. Os tratamentos constaram das seguintes alturas de corte: ao nível do solo (T1), a 0,30 m (T2), a 0,60 m (T3) e a 1,20 metros do nível do solo (T4). Quantificou-se a biomassa da parte aérea de oito plantas de cada bloco, no corte inicial, em janeiro de 2008, e nos meses de março, maio e setembro de 2008, janeiro, março, maio e setembro de 2009, sendo a última determinação realizada em janeiro de 2010. Realizaram-se análises dos teores de nitrogênio (N) do tecido vegetal seco e moído; para tanto, o material foi levado para estufa, a $65^{\circ} \mathrm{C}$, por 48 horas.

Ao término da avaliação da rebrota, na mesma área, avaliou-se a decomposição e liberação de nutrientes da parte aérea de flemingia. Colocaram-se bolsas de decomposição, chamadas de "litter bags", sobre a superfície do solo, no dia 27 de janeiro de 2010, considerado como tempo inicial $\left(\mathrm{T}_{0}\right)$. Adotaram-se três tratamentos: caule ( $\left.\mathrm{T} 1\right)$, folha (T2) e (caule+folha) (T3), em uma razão de 1:1. Esses resíduos da parte aérea foram inseridos nos "litter bags", com dimensão de $25 \mathrm{~cm}$ x $25 \mathrm{~cm}$, com abertura de malha de $4 \mathrm{~mm}$, onde as amostras foram acondicionadas (Anderson \& Ingram, 1993). Inseriram-se, nas bolsas, $50 \mathrm{~g}$ do material vegetal, equivalente, em média, a 3,0 $\mathrm{Mg} \mathrm{MS} \mathrm{ha}^{-1}$. Essas bolsas foram distribuídas na superfície do solo e fixadas com um grampo de arame, evitando-se sua movimentação. O monitoramento da decomposição e da liberação de nutrientes foi realizado por meio de coletas, aos 2, 5, 10, $15,30,60,90,120$ e 150 dias, após a inclusão das bolsas na superfície do solo. Em cada uma dessas coletas, determinaram-se os pesos e os teores dos nutrientes: N, P, K, Ca e Mg. Os valores obtidos foram transformados em percentagem relativa à massa e aos teores de nutrientes do início da decomposição $\left(\mathrm{T}_{\mathrm{o}}\right)$.

Determinaram-se as taxas de decomposição da biomassa e de liberação de nutrientes, para cada um dos tratamentos, utilizando-se o modelo matemático exponencial, descrito por Thomas \& Asakawa, (1993): C $=\mathrm{Coe}^{-\mathrm{kt}}$, em que $\mathrm{C}$ é a quantidade de biomassa seca, ou nutrientes remanescentes, depois de um período de tempo t, em dias; Co refere-se à quantidade de matéria seca no início da decomposição; k é a constante de decomposição. O tempo de meia vida $\left(\mathrm{T}_{1 / 2}\right)$, ou seja, o tempo necessário para que ocorra metade da decomposição ou a metade dos nutrientes sejam liberados da biomassa vegetal existente, foi calculado a partir dos valores de $\mathrm{k}$ do modelo matemático, em que: $\mathrm{T}_{1 / 2}=\operatorname{Ln} 0,5 \mathrm{k}^{-1}$. 


\section{RESULTADOS E DISCUSSÃO}

Os meses de janeiro e fevereiro de 2008 e 2009 foram mais chuvosos, ao passo que, em 2010 e 2011, menos chuvosos (Figura 1). Em 2009, as chuvas iniciaram-se mais cedo, em agosto, o que refletiu na produção de biomassa. A avaliação da rebrota de flemingia revelou uma produção de biomassa seca pouco maior que $15 \mathrm{Mg} \mathrm{ha}^{-1} \mathrm{de} \mathrm{MS,}$ em três cortes realizados no ano de 2008, somando um acúmulo de $365 \mathrm{~kg} \mathrm{ha}^{-1}$ de N, em três cortes, para a altura de 1,2 m do nível do solo, tratamento este que apresentou melhor desempenho na produção de biomassa. Para este mesmo tratamento, os quatro cortes realizados no segundo ano de avaliação, ou seja, em 2009, foi observado o mesmo padrão na produção de biomassa. A produção do primeiro ano de cortes, todavia, foi superada, chegandose a $18 \mathrm{Mg} \mathrm{ha}^{-1} \mathrm{MS}$. Com somente um corte realizado em 2010, a produção de biomassa chegou a $6 \mathrm{Mg} \mathrm{ha}^{-1} \mathrm{MS}$ (Figura 2A).

A primeira avaliação da rebrota, após aplicação dos tratamentos, revelou alta produção de biomassa no corte a 1,2 m. O rendimento chegou próximo a $1,5 \mathrm{Mg} \mathrm{ha}^{-1} \mathrm{de}$ caules e 2,4 $\mathrm{Mg} \mathrm{ha}^{-1} \mathrm{de}$ folhas, superando o tratamento de corte a $0,6 \mathrm{~m}$, em que a soma de matéria seca de caules e folhas chegou a 1,5 $\mathrm{Mg} \mathrm{ha}^{-1}$, seguido do tratamento de corte a $0,3 \mathrm{~m}\left(1,0 \mathrm{Mg} \mathrm{ha}^{-1}\right)$ e corte a $0,0 \mathrm{~m}\left(0,47 \mathrm{Mg} \mathrm{ha}^{-1}\right)$ (Figura 2A).

$\mathrm{Na}$ avaliação da segunda rebrota, observou-se rendimento de biomassa aérea total (caule e folha) de $2,8 \mathrm{Mg}$ $\mathrm{ha}^{-1}$, para os cortes a 1,2 m (Figura 2A). Evidenciou-se que, na medida em que o corte foi realizado mais próximo à superfície do solo, menor foi a produção de biomassa da rebrota, revelando o baixo estoque de reservas, principalmente no tratamento em que o corte é drástico, bem ao nível do solo, eliminando-se toda a parte aérea da planta. O corte realizado a $0,0 \mathrm{~m}$ mostrou um menor desempenho na produção de biomassa, em relação ao dos demais tratamentos.

Na terceira rebrota, a mesma tendência foi observada, em relação à produção de biomassa, pois a maior altura de corte $(1,2 \mathrm{~m})$ proporcionou maior produção, seguida dos demais tratamentos, que diferiram entre si, exceto o do corte realizado a 0,3 metros, em relação ao corte a 0,0 metros do nível do solo. O corte a $0,6 \mathrm{~m}$ apresentou produção de biomassa de 2,6 $\mathrm{Mg} \mathrm{ha}^{-1}$, próxima à do corte mais alto, que chegou a 3,4 Mg ha-1 (Figura 2A). Em todos os tratamentos, observou-se que o intervalo entre cortes adotado neste estudo, proporcionou crescimento. $\mathrm{O}$ corte da biomassa aérea, constituída por caules e folhas tenras e verdes, pode
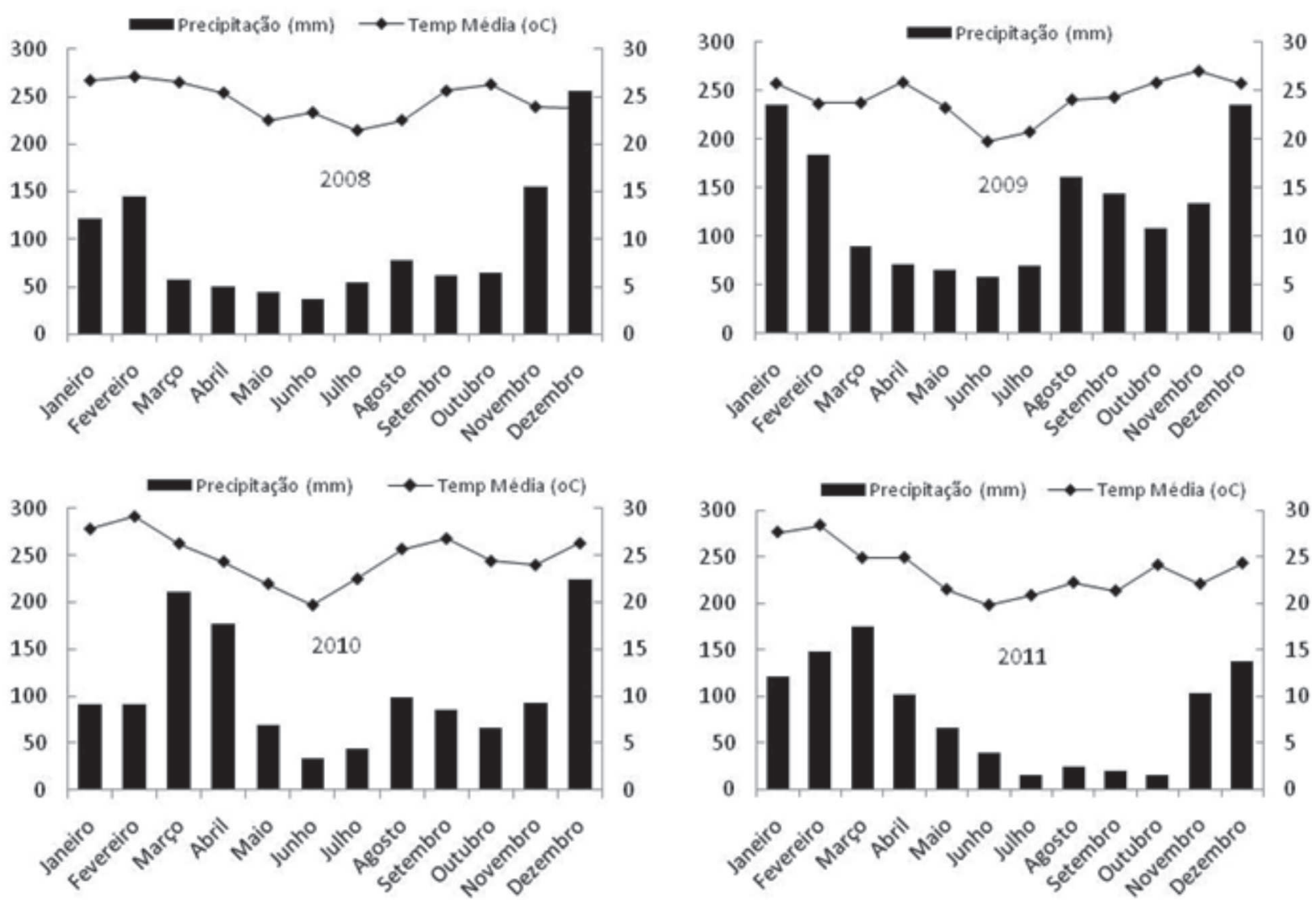

Figura 1. Variação anual da precipitação pluviométrica $(\mathrm{mm})$ e temperatura média do $\operatorname{ar}\left({ }^{\circ} \mathrm{C}\right)$, durante o período do experimento. (Fonte: www.inmet.gov.br/sonabra/maps/automaticas.php: Seropédica, KM 47). 
influenciar a taxa de decomposição e disponibilidade de elementos para as culturas em consórcio.

Para a quarta avaliação, o corte a 1,2 m produziu $6,0 \mathrm{Mg}$ $\mathrm{ha}^{-1}$ de biomassa aérea seca, mostrando efeito superior ao dos demais tratamentos. Para o tratamento de corte a $0,6 \mathrm{~m}$, a produção foi próxima de $4,8 \mathrm{Mg} \mathrm{ha}^{-1}$, e o corte a $0,3 \mathrm{~m}$ proporcionou $2,8 \mathrm{Mg} \mathrm{ha}^{-1}$, seguido do corte a $0,0 \mathrm{~m}$, que se aproximou de 1,0 $\mathrm{Mg} \mathrm{ha}^{-1}$ (Figura 2A). A elevada produção de biomassa foi favorecida pela retomada das águas pluviais, caracterizando um período chuvoso na região.

No quinto corte, realizado em março de 2009, encontraram-se valores próximos aos observados no corte anterior, realizado no mês de janeiro do mesmo ano. Para o corte a 1,2 m, a produção de biomassa foi de 6,0 Mg MS ha $^{-1}$; para o corte a $0,6 \mathrm{~m}$, a produção foi de $4,5 \mathrm{Mg} \mathrm{MS}$ ha $^{-1}$; no corte a $0,3 \mathrm{~m}$, o valor foi de $3,6 \mathrm{Mg} \mathrm{MS} \mathrm{ha}^{-1} \mathrm{e}$, para o corte a $0,0 \mathrm{~m}$, a produção foi de $2,5 \mathrm{Mg} \mathrm{MS} \mathrm{ha}^{-1}$ (Figura 2A). Houve uma queda na produção de matéria seca, no sexto corte . Esse fato pode ser explicado, principalmente, pelo déficit hídrico que se estabeleceu a partir do mês de abril, caracterizando o início do período mais seco do ano. Para maior altura de corte, a produção de biomassa aérea foi de 3,0 Mg MS ha-1; para o corte a 0,6 m, valor próximo de 2,0 $\mathrm{Mg} \mathrm{MS} \mathrm{ha}^{-1}$; no corte a 0,3 , m, a biomassa produzida foi de 1,0 $\mathrm{Mg} \mathrm{MS} \mathrm{ha}^{-1}$, seguida de $0,3 \mathrm{Mg} \mathrm{MS}$ $\mathrm{ha}^{-1}$ para o corte a $0,0 \mathrm{~m}$ do nível do solo (Figura 2A).

Observa-se que os valores de produção de biomassa seca diferem pouco do sexto para o sétimo cortes. Destaquem-se os valores encontrados: para o corte a 1,2 m biomassa de 3,1 $\mathrm{Mg} \mathrm{MS} \mathrm{ha}^{-1}$, seguido de 2,0 Mg MS ha-1, para o corte a $0,6 \mathrm{~m}$; no tratamento com corte $0,3 \mathrm{~m}$, o valor foi de $0,7 \mathrm{Mg} \mathrm{MS} \mathrm{ha}^{-1} \mathrm{e}$, para o corte a $0,0 \mathrm{~m}$, o valor chegou a $0,3 \mathrm{Mg} \mathrm{MS} \mathrm{ha-1}$, próximo ao do corte anterior (Figura 2A). Nota-se que, nos cortes realizados nos períodos mais secos, a produção de biomassa é diminuída, quando se compara com a dos períodos com maior disponibilidade hídrica. Todavia, as plantas permanecem ver- des até mesmo nos períodos secos do ano, mostrando sua capacidade de adaptação a ambientes com baixa disponibilidade hídrica (Salmi et al., 2013).

A retomada da elevada produção de biomassa da espécie pode ser observada, novamente, na realização do oitavo e último corte avaliado no experimento, e refere-se ao mês de janeiro de 2010, cuja produção chegou a 5,8 Mg $\mathrm{MS} \mathrm{ha}^{-1}$, no corte a 1,2 m, com elevado número de ramificações, com boa produção de folhas e apresentando caules tenros. Para o corte a 0,6 m, o valor foi de 4,7 Mg MS ha $^{-1}$. Quantificou-se uma elevada produção de matéria seca para o corte a $0,3 \mathrm{~m}\left(3,6 \mathrm{Mg} \mathrm{MS} \mathrm{ha}^{-1}\right)$, seguido de $1,1 \mathrm{Mg}$ $\mathrm{MS} \mathrm{ha}^{-1}$, para o corte ao nível do solo (Figura 2A).

Notadamente, a espécie apresenta excelente capacidade de se ramificar e alto vigor de rebrota, com elevada produção de biomassa seca em cortes sucessivos. Essa característica é de fundamental importância nos sistemas de cultivos que utilizam espécies arbustivas, como por exemplo, o cultivo em aleias (Friday \& Fownes, 2002), pois possibilita que essas plantas sejam podadas frequentemente, a fim de aportar grande quantidade de material orgânico, favorecendo o desenvolvimento das culturas consorciadas.

Quanto à acumulação de N, observou-se uma proporcionalidade com a produção de biomassa, cujos valores mais elevados são detectados nos tratamentos que apresentaram maior produção de biomassa na parte aérea de flemingia. Todavia, os teores mais elevados de $\mathrm{N}$ foram encontrados nos meses de maio de 2008, maio de 2009 e setembro de 2009 (Tabela 1). Essas épocas caracterizaram-se por um período de precipitação baixa, coincidindo com o final do período chuvoso da região, embora, no mês de setembro de 2009 tivesse ocorrido um volume maior de chuvas, indicando o reinício do período chuvoso na região. Assim como a produção de biomassa foi mais elevada quando as plantas foram submetidas ao corte a 1,2 m do nível do solo, seguida da produção do corte
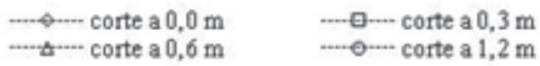

A

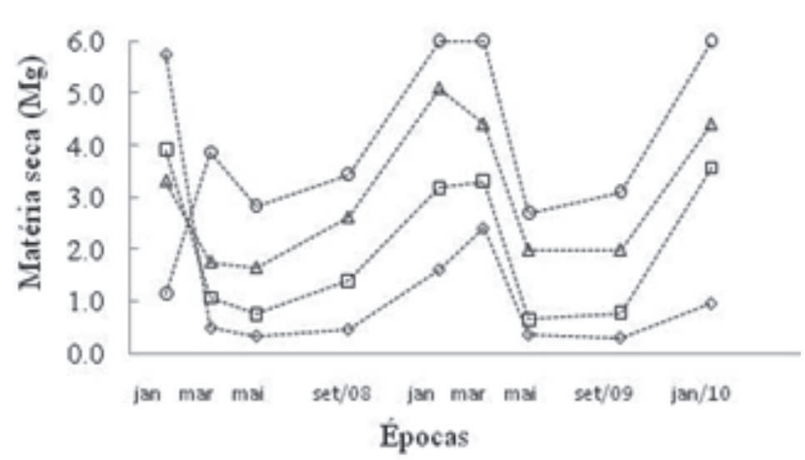

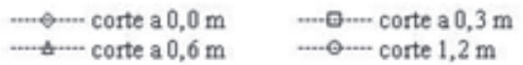

B

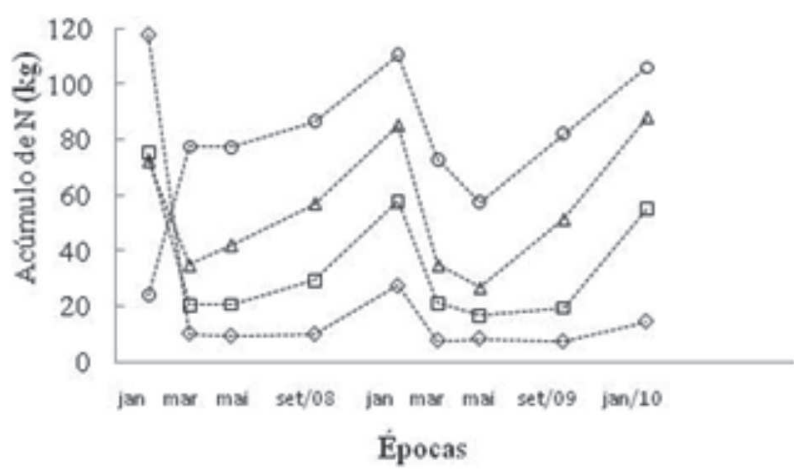

Figura 2. Evolução da produção de matéria seca (A) e acumulação de nitrogênio (B) da parte aérea de Flemingia macrophylla em diferentes épocas e alturas de corte.

Rev. Ceres, Viçosa, v. 60, n.5, p. 735-743, set/out, 2013 
a $0,6 \mathrm{~m}$, depois a $0,3 \mathrm{~m}$ e, enfim, à do corte a $0,0 \mathrm{~m}$, que produziu menor quantidade de biomassa nas diferentes épocas avaliadas (Figura 2B). A acumulação total de nitrogênio, somando-se o corte inicial para estabelecimento das alturas de corte, mais os oito cortes realizados durante dois anos de avaliação, chegou a $211 \mathrm{~kg} \mathrm{~N}$ ha, para o T1 (corte a $0,0 \mathrm{~m}), 329 \mathrm{~kg} \mathrm{~N}$ ha, para o T2 (corte a 0,3 m), $691 \mathrm{~kg} \mathrm{~N}$ ha, para o T3 (corte a 0,6 m) e $804 \mathrm{~kg} \mathrm{~N}$ ha, para o T4 (corte a 1,2 m) (Tabela 2). A produção de biomassa aérea foi maior nas épocas mais chuvosas, para todos os tratamentos, sendo que em janeiro de 2009 não houve diferença significativa entre o corte a 1,2 m e o corte a 0,6 $\mathrm{m}$. Essa elevada produção de biomassa refletiu-se em maior acúmulo de nitrogênio, principalmente nos meses de setembro de 2008, janeiro de 2009, setembro de 2009 e janeiro 2010 (Figura 2B). Isso mostra a excelente capacidade de recuperação, na produção de biomassa da espécie, apresentando altos teores de nitrogênio em sua biomassa, proporcionando elevado acúmulo desse nutriente na parte aérea de flemingia. Destaca-se, então, o alto potencial dessa leguminosa perene, como alternativa de fonte nutricional para o fornecimento de nutrientes na forma de adubo verde.
A constante de decomposição (k) e o tempo de meia vida $\left(\mathrm{T}_{1 / 2}\right)$ da matéria seca da parte aérea de flemingia variaram em decorrência dos diferentes tratamentos. A decomposição do caule segue o mesmo padrão da decomposição da folha e da combinação (caule+folha), na proporção de 1:1. O tempo de meia vida $\left(\mathrm{T}_{1 / 2}\right)$ da decomposição foi de 91, 95 e 97 dias para folha, caule e caule+folha, respectivamente (Tabela 3 ). Nota-se uma ligeira redução do tempo de meia vida de folha, em quatro e seis dias, em relação a caule e caule+folha. Henrot \& Brussard, (1997) encontraram um tempo de meia vida pouco menor para flemingia, de 78 dias, quando a quantidade de biomassa foi de $2 \mathrm{Mg} \mathrm{MS} \mathrm{ha}^{-1}$ em "litter bags", demonstrando uma decomposição ligeiramente mais rápida em relação à verificada neste estudo. Porém, De Costa \& Ataputto, (2001) relatam estudo em que flemingia, após 84 dias, apresentava $69 \%$ de biomassa remanescente, sendo que a decomposição de caule foi mais lenta que a de folha, apresentando $\mathrm{T}_{1 / 2}$ médio de 20,7 semanas, correspondente a 145 dias. Observou-se que, após 30 dias do início do estudo da decomposição da biomassa, o material remanescente de flemingia era de $76,5 \%$, para folha, $86,5 \%$ para caule e $88,5 \%$ para caule+folha. Embora com decomposi-

Tabela 1. Teores de nitrogênio da parte aérea de flemingia nas oito épocas para as três alturas de corte, aplicadas durante a condução do experimento

\begin{tabular}{lcccccccc}
\hline & \multicolumn{7}{c}{ Épocas de corte } \\
\cline { 2 - 9 } Trat. & mar/08 & mai/08 & set/08 & jan/09 & mar/09 & mai/09 & set/09 & jan/10 \\
\cline { 2 - 9 } & & \multicolumn{7}{c}{ Teor de nitrogênio $\left(\mathbf{g}^{\prime} \cdot \mathbf{k g}^{-1}\right)$} \\
\hline $0,0 \mathrm{~m}$ & $20,5 \mathrm{aC} *$ & $28,5 \mathrm{aA}$ & $21,6 \mathrm{aC}$ & $18,1 \mathrm{aD}$ & $21,7 \mathrm{aC}$ & $23,7 \mathrm{bB}$ & $24,7 \mathrm{aB}$ & $15,6 \mathrm{aE}$ \\
$0,3 \mathrm{~m}$ & $19,2 \mathrm{aB}$ & $27,6 \mathrm{aA}$ & $21,0 \mathrm{aB}$ & $18,2 \mathrm{aB}$ & $21,9 \mathrm{aB}$ & $25,3 \mathrm{bA}$ & $25,1 \mathrm{aA}$ & $15,8 \mathrm{aC}$ \\
$0,6 \mathrm{~m}$ & $19,9 \mathrm{aB}$ & $25,7 \mathrm{abA}$ & $21,7 \mathrm{aB}$ & $17,6 \mathrm{aC}$ & $23,3 \mathrm{aB}$ & $27,1 \mathrm{aA}$ & $26,0 \mathrm{aA}$ & $16,3 \mathrm{aC}$ \\
$1,2 \mathrm{~m}$ & $19,9 \mathrm{aC}$ & $25,0 \mathrm{bA}$ & $20,6 \mathrm{aC}$ & $18,1 \mathrm{aD}$ & $22,7 \mathrm{aC}$ & $25,4 \mathrm{bA}$ & $26,7 \mathrm{aA}$ & $17,8 \mathrm{aD}$ \\
\hline
\end{tabular}

* Valores seguidos de mesma letra minúscula, na coluna, não diferem entre as alturas de corte e, mesma letra maiúscula, na linha, não diferem entre as épocas a $5 \%$ de probabilidade pelo teste de Tukey.

Tabela 2. Quantidade de matéria seca e nitrogênio, acumulados em oito cortes realizados em diferentes alturas de corte

\begin{tabular}{lcrrrr}
\hline \multirow{2}{*}{ Anos } & Acumulação & \multicolumn{4}{c}{ Altura de corte } \\
\cline { 3 - 6 } & & $\mathbf{0 , 0} \mathbf{~ m}$ & $\mathbf{0 , 3} \mathbf{~ m}$ & $\mathbf{0 , 6} \mathbf{~ m}$ & $\mathbf{1 , 2} \mathbf{~ m}$ \\
\hline \multirow{2}{*}{ Corte inicial } & Matéria Seca $\left(\mathrm{MS} \mathrm{ha}^{-1}\right)$ & $5,76 \mathrm{a}^{(1)}$ & $3,91 \mathrm{~b}$ & $3,31 \mathrm{c}$ & $1,14 \mathrm{~d}$ \\
& Nitrogênio $\left(\mathrm{N} \mathrm{ha}^{-1}\right)$ & $118,08 \mathrm{a}$ & $90,05 \mathrm{~b}$ & $72,16 \mathrm{c}$ & $23,83 \mathrm{~d}$ \\
\cline { 2 - 6 } 2008 & Matéria Seca & $1,59 \mathrm{~d}$ & $3,18 \mathrm{c}$ & $5,99 \mathrm{~b}$ & $10,13 \mathrm{a}$ \\
& Nitrogênio & $28,98 \mathrm{~d}$ & $70,13 \mathrm{c}$ & $133,88 \mathrm{~b}$ & $351,76 \mathrm{a}$ \\
\hline \multirow{2}{*}{2009} & Matéria Seca & $7,71 \mathrm{c}$ & $7,94 \mathrm{c}$ & $14,89 \mathrm{~b}$ & $17,43 \mathrm{a}$ \\
& Nitrogênio & $50,10 \mathrm{c}$ & $114,17 \mathrm{~b}$ & $397,75 \mathrm{a}$ & $323,08 \mathrm{ab}$ \\
\hline \multirow{2}{*}{$2010^{*}$} & Matéria Seca & $0,95 \mathrm{c}$ & $3,56 \mathrm{~b}$ & $5,56 \mathrm{a}$ & $5,95 \mathrm{a}$ \\
& Nitrogênio & $14,44 \mathrm{~d}$ & $55,04 \mathrm{c}$ & $87,71 \mathrm{~b}$ & $105,88 \mathrm{a}$ \\
\hline & Total MS $\left(\mathrm{Mg} \mathrm{ha}^{-1}\right)$ & $16,01 \mathrm{~d}$ & $18,59 \mathrm{c}$ & $29,75 \mathrm{~b}$ & $34,65 \mathrm{a}$ \\
\hline
\end{tabular}

\footnotetext{
(1) Valores seguidos de mesma letra minúscula, na linha, não diferem entre si a $5 \%$ de probabilidade pelo teste de Tuckey.

* somente um corte.
} 
ção inicial mais lenta em relação à da folha e caule, a relação caule+folha apresentou menor quantidade de material nos "litter bags" por ocasião da última coleta, aos 150 dias, com 55,4\% de biomassa remanescente. Para folhas, a biomassa remanescente foi de $58,6 \%$ e para caules, $60,5 \%$, nesse mesmo tempo (Figura $3 \mathrm{~A}$ ). Os $\mathrm{T}_{1 / 2}$ encontrados são bem superiores aos de outros adubos verdes estudados no mesmo local: Crotalaria juncea, 40 dias, Mucuna cinerea, 38 dias, Glricidia sepium, 21 dias (Coelho et al., 2006; Silva et al., 2007).

As concentrações iniciais de nutrientes na biomassa da parte aérea de flemingia são apresentadas na Tabela 3. A dinâmica de mineralização de N, P, K, Ca e Mg, para caules, folhas e caules + folhas são similares. A decomposição foi relativamente lenta, quando comparada com a de outras espécies (Salmi et al., 2006), o que pode ser causado pela concentração alta de taninos e ligninas presentes no tecido dessa espécie (Stürm et al., 2007). Neste estudo, observa-se maior conteúdo de nitrogênio $(\mathrm{N})$ nas folhas, seguido do conteúdo da combinação caules + folhas (1:1) e, depois, nos caules. O fósforo (P), potássio $(\mathrm{K})$, cálcio $(\mathrm{Ca})$ e magnésio $(\mathrm{Mg})$ apresentaram esse mesmo comportamento, quanto ao conteúdo: folhas > caules+ folhas +caules (Tabela 3 ).

$\mathrm{O}$ nitrogênio $(\mathrm{N})$ remanescente, expresso como percentagem do $\mathrm{N}$ inicial, seguiu o mesmo comportamento observado para a biomassa remanescente, apresentando, no início da decomposição, liberação mais acelerada, detectada nas primeiras coletas, seguida de uma fase de liberação mais lenta, o que ocorreu em todos os tratamentos. Em vista disso, o ajuste dos dados adequou-se ao modelo exponencial (Figura 3B). O tempo de meia vida $\left(\mathrm{T}_{1 / 2}\right)$ para liberação deste elemento nas folhas foi de 116 dias, nos caules, de 102 dias e, na relação caules + folhas, de 99 dias (Tabela 4). A liberação de N ocorreu de forma mais pronunciada até os 15 dias após inclusão das bolsas "litter bags", no campo, atingindo $26,2 \mathrm{~kg} \mathrm{~N}^{-1}$, equivalente a $32 \%$ do $\mathrm{N}$ acumulado nas folhas. Nos caules, a liberação foi ligeiramente mais lenta, para esse mesmo período, chegando a $14 \%$, equivalente a $3,65 \mathrm{~kg} \mathrm{~N} \mathrm{ha}^{-1} \mathrm{e}$, para a combinação de caules mais folhas, esse valor chegou a $14,5 \mathrm{~kg} \mathrm{~N} \mathrm{ha}^{-1}$, equivalendo a $22 \%$ da decomposição. Ao final deste estudo, portanto, aos 150 dias, folhas apresentavam $36 \%$ de nitrogênio remanescente; caules,
$42 \%$ e, caules + folhas, 28\% (Figura 3B). Zingore et al., (2003) verificaram incremento de $1,8 \mathrm{mg} \cdot \mathrm{kg}^{-1} \mathrm{de} \mathrm{N}$, após dez semanas da incorporação de $5 \mathrm{Mg} \mathrm{ha}^{-1}$ de flemingia, em um solo arenoso do Zimbabwe. A liberação lenta desse nutriente, acompanhada de uma decomposição retardada, pode minimizar processos de lixiviação, assegurando um incremento considerável de matéria orgânica rica em N, em longo prazo, trazendo melhorias na fertilidade dos solos. Förster et al., (2009) encontraram aumento na biomassa microbiana e nos teores de nitrato, em solos com biomassa de flemingia.

Aos 15 dias, os três tratamentos apresentaram taxas de liberação de fósforo semelhantes, sendo que, para as folhas, a quantidade remanescente desse elemento foi de $71 \%$; para caules+folhas, de $72 \%$ e, para caules, de $76 \%$. Na última coleta, realizada aos 150 dias, observou-se que, para folhas, havia $35 \%$ deste nutriente remanescente na biomassa; para caules, $41 \%$ e, para caules + folhas, 33\% (Figura 3C). $\mathrm{O} \mathrm{T}_{1 / 2}$ de $\mathrm{P}$ nas folhas foi de 81 dias; para caules, de 80 dias; para caules + folhas, de 67 dias (Tabela 4). De Costa \& Ataputtu (2001) encontraram, para folhas, um $\mathrm{T}_{1 / 2}$ de 25,5 dias e, para caules, $\mathrm{T}_{1 / 2}$ de 33 dias, valores mais baixos que os encontrados neste estudo. A liberação de fósforo pelas folhas foi mais lenta no início da decomposição, em relação à dos demais tratamentos, que seguiram um mesmo padrão que a liberação de nitrogênio (Figura 3B). A espécie flemingia apresenta um sistema radicular profundo e profuso, mostrando-se altamente capaz de absorver e ciclar esse elemento.

A liberação de potássio nas folhas foi a mais lenta entre as dos outros tratamentos, até os 15 dias, verificando-se $75 \%$ deste elemento remanescente na biomassa; para os caules, de $60 \%$ e, para caules + folhas, de $58 \%$. Embora, aos 150 dias, a diferença do percentual remanescente de $\mathrm{K}$ entre os tratamentos tenha sido pequena, sendo $15 \%$ nas folhas, $12 \%$, nos caules, e $10 \%$, em caules + folhas (Figura 3D). $\mathrm{O} \mathrm{T}_{1 / 2}$ na liberação de $\mathrm{K}$ para folhas foi de 21 dias, de 40 dias, para caules e de 19 dias para caules+folhas (Tabela 4). Estudos de De Costa \& Ataputtu (2001) relatam um $\mathrm{T}_{1 / 2}$ de 17,1 dias para folhas e de 40,4 dias para caules, no Sri Lanka, valores que corroboram os deste estudo. O potássio está presente como cátion que se move livremente no fluído celular. Quando as membra-

Tabela 3. Concentração inicial de nutrientes na folha, caule e caule + folha da parte aérea de flemingia inserida nas bolsas "litter bags"

\begin{tabular}{lccccc}
\hline \multirow{2}{*}{ Tratamentos } & $\mathbf{N}$ & $\mathbf{P}$ & $\mathbf{K}$ & $\mathbf{C a}$ & $\mathbf{M g}$ \\
\cline { 2 - 6 } & \multicolumn{5}{c}{ g/kg de matéria seca } \\
\hline Folha & $27,3 \mathrm{a}^{*}$ & $2,23 \mathrm{a}$ & $7,81 \mathrm{a}$ & $8,50 \mathrm{a}$ & $2,90 \mathrm{a}$ \\
Caule & $8,7 \mathrm{~b}$ & $1,76 \mathrm{~b}$ & $5,13 \mathrm{c}$ & $6,96 \mathrm{~b}$ & $2,26 \mathrm{~b}$ \\
Caule+Folha & $22,0 \mathrm{ab}$ & $2,09 \mathrm{ab}$ & $6,81 \mathrm{~b}$ & $8,24 \mathrm{ab}$ & $2,64 \mathrm{ab}$ \\
\hline
\end{tabular}

* Valores seguidos de mesma letra minúscula, na linha, não diferem entre si a 5\% de probabilidade pelo teste de Tuckey.

Rev. Ceres, Viçosa, v. 60, n.5, p. 735-743, set/out, 2013 
nas desintegram-se, o potássio é facilmente lavado do material orgânico. Esse nutriente não faz parte de nenhum composto orgânico e, portanto, é mais facilmente lavado por ocasião da decomposição da matéria orgânica (Bayer \& Mielniczuk, 2008).

A liberação de cálcio nas folhas mostra-se ligeiramente mais lenta que a dos caules e de caules + folhas, embora o teor inicial de cálcio na biomassa aérea de flemingia tenha diferido estatisticamente, seguindo a seguinte ordem: folhas $\left(8,5 \mathrm{~g} \mathrm{~kg}^{-1}\right)>$ caules + folhas $\left(8,2 \mathrm{~g} \mathrm{~kg}^{-1}\right)>$ caules $(6,9 \mathrm{~g}$ $\mathrm{kg}^{-1}$ ) (Tabela 3). $\mathrm{O} \mathrm{T}_{1 / 2}$, na liberação de Ca das folhas foi de 235 dias; de caules, de 163 dias; de caules + folhas, de 168 dias (Tabela 4). Aos 30 dias após iniciado o experimento, a percentagem de material remanescente nas folhas foi de $80,3 \%$, enquanto, em caules, foi de $73,9 \%$ e, em caules + folhas, de 72,2\%. Pode-se observar que, ao final do experimento, portanto, aos 150 dias, a combinação caules + folhas havia liberado maior quantidade desse elemento no sistema, com $46,2 \%$, seguida de caules, com $45,5 \%$, e de folhas, com 37,4\% de cálcio liberado (Figura 3E).

A liberação de magnésio mostra comportamento similar à liberação de cálcio. Aos 15 dias, havia $75 \%$ de magnésio remanescente nas folhas; 76,8\%, em caules, e $85 \%$, em caules + folhas. As quantidades remanescentes aproximaram-se, nos diferentes tratamentos, aos 120 dias, apresentando valores de 54,2\% para folhas; para caules, valor pouco mais alto, com $57,3 \%$ e, para caules + folhas, de 54,3\% (Figura 3F). O T $1 / 2$ na liberação de magnésio foi de 136 dias, para folhas; de 159, para caules e de 152 dias para a combinação caules + folhas (Tabela 4).
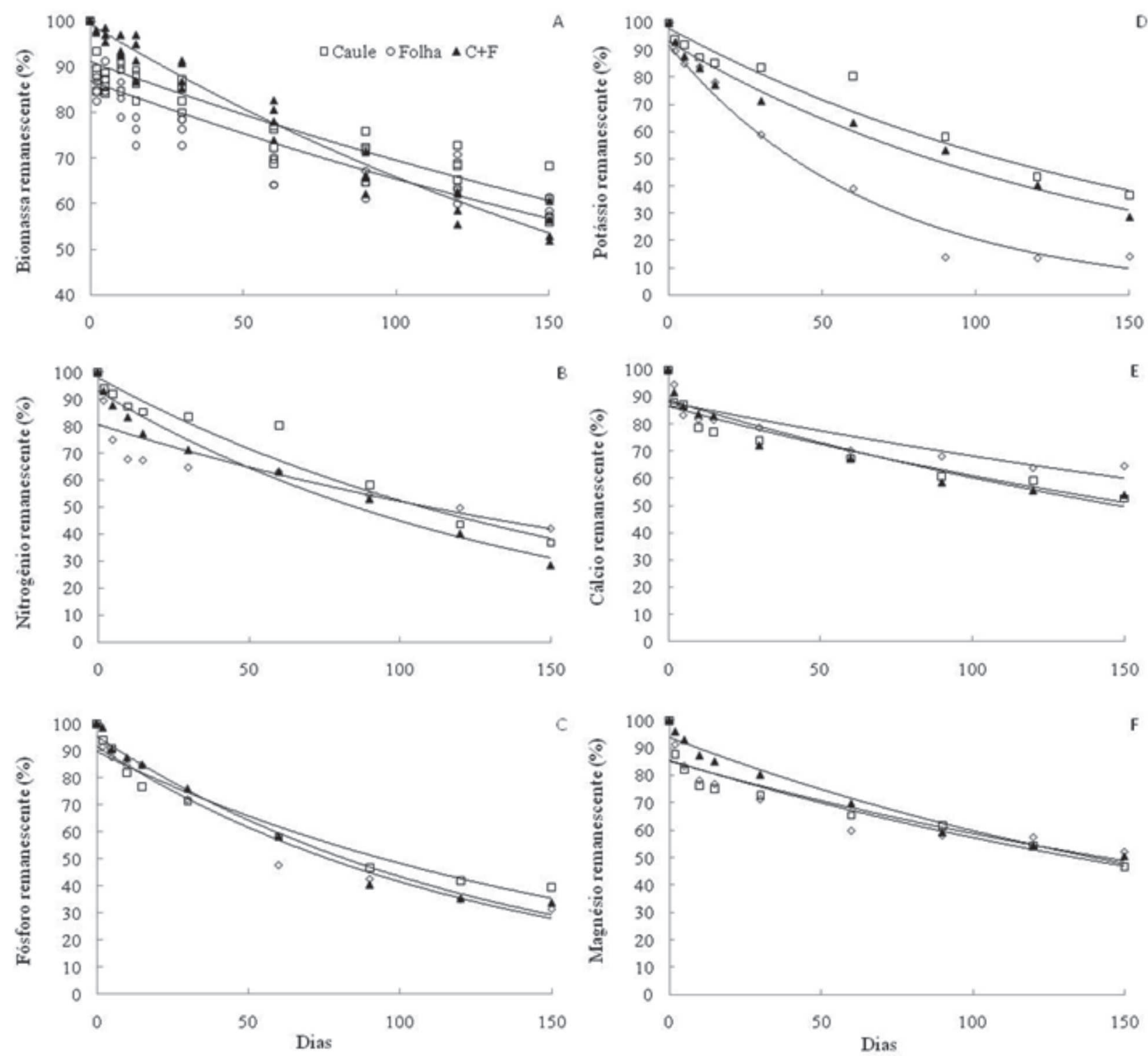

Figura 3. Taxa de decomposição (A) e mineralização dos nutrientes: (B) nitrogênio, (C) fósforo, (D) potássio, (E) cálcio e (F) magnésio da biomassa aérea de Flemingia macrophylla ao longo de cinco meses de condução do experimento. 
Tabela 4. Parâmetros da função $\mathrm{C}=\mathrm{Coe}{ }^{-k t}$, tempo de meia vida $\left(\mathrm{T}_{1 / 2}\right)$ da taxa de liberação de $\mathrm{N}, \mathrm{P}, \mathrm{K}, \mathrm{Ca}$ e $\mathrm{Mg}$ contidos nos resíduos vegetais de flemingia

\begin{tabular}{|c|c|c|c|c|}
\hline \multirow{3}{*}{ Tratamentos } & \multicolumn{4}{|c|}{ Parâmetros da equação ${ }^{(1)}$ de decomposição e liberação de nutrientes } \\
\hline & \multicolumn{4}{|c|}{ Decomposição biomassa } \\
\hline & $K^{(2)}\left(g \cdot g^{-1} \cdot\right.$ dia $\left.^{-1}\right)$ & $T_{1 / 2}^{(3)}$ (dias) & Equação & Coef. de determ. $\left(\mathbf{R}^{2}\right)$ \\
\hline Caule & 0.0073 & 95 & $y=91,137 \mathrm{e}^{-0,0027 x}$ & 0.85 \\
\hline Folha & 0.0078 & 91 & $\mathrm{y}=86,907 \mathrm{e}^{-0,003 \mathrm{x}}$ & 0.80 \\
\hline \multirow[t]{2}{*}{ Caule + Folha } & 0.0072 & 97 & $y=99,37 e^{-0,004 x}$ & 0.96 \\
\hline & \multicolumn{4}{|c|}{ Nitrogênio (N) } \\
\hline Caule & 0.0068 & 102 & $\mathrm{y}=92,707 \mathrm{e}^{-0,073 x}$ & 0.94 \\
\hline Folha & 0.0060 & 116 & $\mathrm{y}=75,870 \mathrm{e}^{-0,015 \mathrm{x}}$ & 0.94 \\
\hline \multirow[t]{2}{*}{ Caule + Folha } & 0.7000 & 99 & $\mathrm{y}=93,061 \mathrm{e}^{-0,007 \mathrm{x}}$ & 0.98 \\
\hline & \multicolumn{4}{|c|}{ Fósforo $(\mathrm{P})$} \\
\hline Caule & 0.0091 & 80 & $\mathrm{y}=141,630 \mathrm{e}^{-0,257 \mathrm{x}}$ & 0.84 \\
\hline Folha & 0.0090 & 81 & $\mathrm{y}=89,784 \mathrm{e}^{-0,006 \mathrm{x}}$ & 0.95 \\
\hline \multirow[t]{2}{*}{ Caule + Folha } & 0.0110 & 67 & $y=91,578 e^{-0,008 x}$ & 0.97 \\
\hline & \multicolumn{4}{|c|}{ Potássio $(\mathrm{K})$} \\
\hline Caule & 0.0178 & 40 & $\mathrm{y}=120,210 \mathrm{e}^{-0,136 \mathrm{x}}$ & 0.89 \\
\hline Folha & 0.0340 & 21 & $\mathrm{y}=97,954 \mathrm{e}^{-0,006 \mathrm{x}}$ & 0.96 \\
\hline \multirow[t]{2}{*}{ Caule + Folha } & 0.0400 & 19 & $y=73,092 e^{-0,016 x}$ & 0.92 \\
\hline & \multicolumn{4}{|c|}{ Cálcio $(\mathrm{Ca})$} \\
\hline Caule & 0.0030 & 235 & $\mathrm{y}=88,053 \mathrm{e}^{-0,003 \mathrm{x}}$ & 0.81 \\
\hline Folha & 0.0040 & 163 & $\mathrm{y}=85,507 \mathrm{e}^{-0,004 \mathrm{x}}$ & 0.91 \\
\hline \multirow[t]{2}{*}{ Caule + Folha } & 0.0040 & 168 & $y=93,840 e^{-0,005 x}$ & 0.97 \\
\hline & \multicolumn{4}{|c|}{ Magnésio (Mg) } \\
\hline Caule & 0.0400 & 159 & $\mathrm{y}=85,193 \mathrm{e}^{-0,004 \mathrm{x}}$ & 0.84 \\
\hline Folha & 0.0050 & 136 & $y=86,498 e^{-0,003 x}$ & 0.89 \\
\hline Caule + Folha & 0.0050 & 152 & $\mathrm{y}=88,822 \mathrm{e}^{-0,004 \mathrm{x}}$ & 0.91 \\
\hline
\end{tabular}

(1) Equação: C=Coe-kt; ${ }^{(2)}$ Constante de decaimento; ${ }^{(3)}$ Tempo de meia vida

\section{CONCLUSÕES}

A espécie apresenta elevada produção de biomassa aérea, rica em nitrogênio, podendo ser podada periodicamente, sendo que a altura de corte influencia no vigor da rebrota. As taxas de decomposição e liberação dos nutrientes da parte aérea de flemingia, tanto para folhas, quanto para caules e caules + folhas, apresentam padrões semelhantes, sendo maior para o K, podendo esta espécie ser considerada de lenta decomposição.

\section{AGRADECIMENTOS}

À Fundação Carlos Chagas de Amparo à Pesquisa do Estado do Rio de Janeiro (FAPERJ), pela concessão da bolsa Cientista do Nosso Estado.

\section{REFERÊNCIAS}

Andersson MS, Schultze-Kraft R, Cansa M, Hincapie' B \& Lascano CE (2006) Morphological, agronomic and forage quality diversity of the Flemingia macrophylla world collection. Field Crops Research, 96:387-406.
Anderson JM \& Ingram JSI (1993) Tropical Soil Biological and Fertility: a handbook of methods. 2.ed. Wallingford, CAB International. 221p.

Bayer C \& Mielniczuk J (2008) Dinâmica e funções da matéria orgânica. In: Santos GA, Silva L, Canellas LP \& Camargo FAO (Eds.) Fundamentos da matéria orgânica do solo: ecossistemas tropicais. $2^{a}$ ed. Porto Alegre, Metrópole. p. $7-18$.

Coelho RA, Silva GTA, Ricci MSF \& Resende AS (2006) Efeito de leguminosa arbórea na nutrição nitrogenada do cafeeiro (Coffea canephora Pierre ex Froenh) consorciado com bananeira em sistema orgânico de produção. Coffe Science, $1: 21-27$.

Costa CHM, Crusciol CAC, Soratto RP \& Ferrari Neto J (2012) Persistência e liberação de macronutrientes e silício da fitomassa de crotalária em função da fragmentação. Bioscience Journal, 28:384-394.

De Costa WAJM \& Atapattu AMLK (2001) Decomposition and nutrient loss from prunings of different contour hedgerow species in tea plantations in the sloping highlands of Sri Lanka. Agroforestry Systems, 51:2010-211.

Embrapa Centro Nacional de Pesquisa de Solos (2006) Sistema brasileiro de classificação de solos. $2^{\mathrm{a}}$ ed. Rio de Janeiro, EmbrapaSPI. 306p.

Rev. Ceres, Viçosa, v. 60, n.5, p. 735-743, set/out, 2013 
Förster B, Garcia M, Höfer H, Morgan E \& Römbke J (2009) Tropical terrestrial model ecosystems for evaluation of soi fauna and leaf litter quality effects on litter consumption, soil microbial biomass and plant growth. Pesquisa Agropecuária Brasileira, 44:1063-1071.

Friday JB \& Fownes JH (2002) Competition for light between hedgerows and maize in alley cropping system in Hawaii, USA. Agroforestry Systems, 55:125-137.

Henrot J \& Brussard L (1997) Determinats of Flemingia congesta and Dactyladenia barteri mulch decomposition in alley-cropping systems in the humid tropics. Plant and Soil, 191:101-107.

Salmi AP, Guerra JGM, Abboud ACS, Lopes HM \& Martelleto MS (2008) Superação da dormência das sementes de Flemingia macrophylla. Seropédica, Embrapa Agrobiologia. (Comunicado Técnico 108).

Salmi AP, Risso IAM, Guerra JGM, Urquiaga S, Araújo AP \& Abboud ACS (2013) Crescimento, acúmulo de nutrientes e fixação biológica de nitrogênio de Flemingia macrophylla. Revista Ceres, 60:79-85
Salmi GP, Salmi AP \& Abboud AC (2006) Dinâmica de decomposição e liberação de nutrientes de genótipos de guandu sob cultivo em aléias. Pesquisa Agropecuária Brasileira, 41:673-678.

Silva GTA, Oliveira WRD, Matos LV, Nóbrega PO, Krainovic PO, Campello EFC \& Resende AS (2007) Correlação entre a composição química e a velocidade de decomposição de plantas para adubação verde visando à elaboração de uma base de dados. Seropédica, Embrapa Agrobiologia. 51p. (Boletim de Pesquisa e Desenvolvimento, 21).

Stürm CD, Tiemann TT, Lascano CE, Kreuzer M \& Hess HD (2007) Nutrient composition and in vitro ruminal fermentation of tropical legumemixtures with costrasting tannin contents. Animal Feed Science and Technology, 138:29-46.

Thomas RJ \& Asakawa NM (1993) Decomposition of leaf litter from tropical forage grasses and legumes. Soil Biology and Biochemistry, 25:1351-1361.

Zingore S, Mafongoya P, Nyamugafata P \& Giller KE (2003) Nitrogen mineralization and maize yields following application of tree prunings to a sandy soil in Zimbabwe. Agroforestry Systems, 57:199-211. 